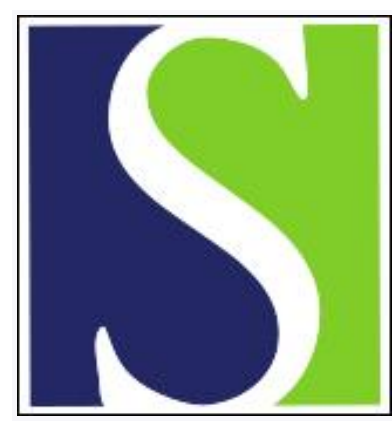

Scand J Work Environ Health 2011;37(3):169-171

https://doi.org/10.5271/sjweh.3161

Published online: 28 Mar 2011, Issue date: May 2011

\title{
What constitutes an unsafe work schedule?
}

by Tucker $\mathrm{P}$

Affiliation: Department of Psychology, Swansea University, Swansea SA2 8PP, United Kingdom. p.t.tucker@swan.ac.uk

Refers to the following texts of the Journal: 2011;37(3):173-185 2010;36(6):458-465 2006;32(3):232-240 1987;13(6):486-492

Key terms: accident; editorial; long working hours; night work; shift work; unsafe work; work schedule; working time

This article in PubMed: www.ncbi.nlm.nih.gov/pubmed/21448551 


\section{What constitutes an unsafe work schedule?}

While the impact of non-standard working hours on worker fatigue and consequent accident risk are commonly acknowledged as a cause for concern, the precise nature of that relationship (eg, what constitutes an "unsafe" work schedule) remain unclear. Methodological weaknesses of some studies and inconsistencies between studies (eg, in terms of methods, definitions, etc) have contributed to the muddying of the waters. Thus the review by Wagstaff \& Lie (1), involving the systematic selection of studies on the basis of high methodological standards, is to be welcomed as a means of shedding some light on the issue. It is the first systematic review (ie, using clearly defined criteria and standardized evaluation in the selection of articles) of work schedules and safety, conducted across all occupational sectors. Increased levels of risk were identified in association with long work shifts, night work, and rotating shifts. There were no indications within the selected studies that either age or gender moderated the effects of work schedules on accident risk.

As the review's authors point out, one of the problems that has afflicted this topic is that many studies have tended to under-specify the design of the schedules being studied. For example, comparisons between day and night work might not specify how many nights were worked consecutively, even though this is known to influence the degree of fatigue and risk associated with night working $(2,3)$. This omission may of course be for very good reasons, for example, because the number of consecutive night shifts varied within the sample. Nevertheless, interpretation of such a comparison's results remains problematic.

Of the three studies of "permanent" night shifts identified by Wagstaff \& Lie, two found no significant increase in risk. However it was not clear in either case whether the "permanent" night workers regularly worked several nights in a row. This is important as the term "permanent night work" is often interpreted as working a whole week of nights, for week after week. Yet, in the study by Gold et al (4), night workers were defined as those working $\geq 8$ night shifts and no day/evening shifts within a month. In the study by Fransen et al (5), it was noted that more than $50 \%$ of the night workers usually worked $<3$ nights per week. (It is also interesting to note that the latter study included sleep difficulties as one of the control variables in their analysis. Given that sleep difficulties are a likely mediator of the relationship between night work and increased risk, this might be viewed as "over-controlling" in the analysis). In contrast to the first two studies, the third study (6), in which permanent night work was defined as "regular night shifts", did find significantly increased risk. Given the question marks over the definitions used in the first two studies, together with the positive finding of the third, there may be reason to doubt that permanent night work is associated with relatively low risk.

The finding that rotating shifts are associated with increased risk begs the question as to which types of rotating shifts were involved in the comparisons with day work. Most research findings tend to favor very rapidly rotating shift systems (ie, ones that involve working only 1-3 consecutive shifts of the same type) over more slowly rotating ones. Moreover, there is some suggestion in the literature, albeit equivocal, that forward rotating shifts may be associated with lower levels of fatigue than backward rotating ones (7). Thus it is possible that the high levels of risk associated with rotating shifts identified in the current review may have been at least partly a function of the particular patterns of rotating shifts examined in the selected studies.

All this suggest that the apparently benign effects of permanent night shifts, relative to rotating shifts, may have been overstated in the current review. While there may be some merit in the suggestion that permanent night shifts could offer some protection through resynchronization, this may only be true for a minority of workers (8). 
Another problem that can affect comparisons between work schedules, as well comparisons between different shifts within a schedule, is that the a priori risk may not be constant across the comparison. That is to say, it can difficult to determine how much of a difference in risk is due to fatigue-related deficits in human performance versus other risk factors (eg, exposure rate, hazard level) that vary concurrently. While many studies of the impact of work schedules suffer from problems such as unknown exposure rates and hazard levels, those selected in the current review were assessed in terms of residual confounding. Hence we can be reasonably confident that this problem will have been at least partly addressed in the current review. However, it will always be difficult to know whether all relevant confounds have been eliminated.

A long work day means that the window of opportunity for rest and recovery before returning to work the next day is reduced. Thus, if another duty is scheduled for the next day, the individual will return to work less recuperated than if the previous work day had been shorter. If this same pattern of long work periods and inadequate recovery is repeated day-after-day, throughout the week, and perhaps also from one week to the next, the result is likely to be a substantial accumulation of fatigue and associated problems. Thus long work duties are particularly problematic when there is inadequate opportunity for regular rest and recovery between duty periods. This highlights the need to consider the length of an individual duty period in light of the accumulated number of hours that are worked during the week. There is an important distinction to be drawn between working several long duty periods in a week (eg, working $\geq 5$ days per week with overtime), such that the overall weekly hours are high and rest opportunities are limited, and compressing the normal weekly work hours into fewer longer duty periods (ie, extending daily work hours). For example, when an increase in risk is reported in the latter stages of extended shifts, it is important to know whether working a single extended shift will produce such an increase, or whether the trend partly reflects accumulated fatigue that results from having limited time-off during the week. Some of the studies cited in the current review do report separately the associations with daily and weekly work hours. However, it is not always clear whether the length of the shift is associated with increased risk independently of the number of hours worked per week. In this regard, it interesting to note an epidemiological study of medical worker's injuries (not included in the current review) which reported that while working $>60$ hours per week was associated with increased risk, working $\geq 12$ hours per day was not (9).

Identifying potential moderators of the relationship between work schedules and risk (eg, age and gender) is fraught with difficulties. As noted by Wagstaff \& Lie (1), selection effects make it difficult to establish how aging interacts with work schedule in the prediction of risk. Regarding gender, it is important to remember that comparisons of the impact that work schedules have on males and females tend to be confounded by a wide range of other factors (eg, occupation, job and working time control, engagement in domestic and caring duties, social class, and marital status). Such confounding makes it impossible to know whether gender differences in the impact of particular work schedules is due to the fact that men and women tend to work in different occupations, have different levels of job control, etc.

Shift work, night work, and long work hours are potentially very important contributing factors to the risk of occupational accidents and injuries. The review by Wagstaff \& Lie provides some useful indications of which aspects of work schedules may be associated with increased risk. However, it also highlights a number of unresolved issues that affect our ability to unambiguously interpret the findings of even the more methodologically rigorous studies in this area. In this respect, hopefully their review will encourage and inform future studies that clarify the issues and expand our understanding of the relationship between work schedule features and accident risk. 


\section{References}

1. Wagstaff AS, Sigstad Lie J-A. Shift and night work and long working hours - a systematic review of safety implications. Scand J Work Environ Health. 2011;37(3):173-185. doi:10.5271/sjweh.3146

2. Folkard S, Tucker P. Shift work, safety and productivity. Occup Med. 2003;53(2): 95-101.

3. Tucker P, Brown M, Dahlgren A, Davies G, Ebden P, Folkard S, et al. The impact of junior doctors' working time arrangements on their fatigue and wellbeing. Scand J Work Environ Health. 2010;36(6):458-65. doi:10.5271/ sjweh.2985.

4. Gold DR, Rogacz S, Bock N, et al. Rotating shift work, sleep, and accidents related to sleepiness in hospital nurses. Am J Public Health. 1992;82:1011-14. doi:10.2105/AJPH.82.7.1011.

5. Fransen M, Wilsmore B, Winstanley J, et al. Shift work and work injury in the New Zealand Blood Donors' Health Study. Occup Environ Med. 2006;63:352-58. doi:10.1136/oem.2005.024398

6. Dembe AE, Erickson JB, Delbos RG, Banks SM. Nonstandard shift schedules and the risk of job-related injuries. Scand J Work Environ Health. 2006;32:232-240. doi:10.5271/sjweh.1004

7. Sallinen M, Kecklund G. Shift work, sleep and sleepiness - differences between shift schedules and systems. Scand J Work Environ Health. 2010;36(2):121-133. doi:10.5271/sjweh.2900

8. Folkard S. Do permanent night workers show circadian adjustment? A review based on the endogenous melatonin rhythm. Chronobiol Int. 2008;25(2):215-24.

9. Dembe AE, Delbos R, Erickson JB. Estimates of injury risks for healthcare personnel working night shifts and long hours. Qual Saf Health Care. 2009;18:336-40.

Philip Tucker, PhD

Department of Psychology,

Swansea University,

Swansea SA2 8PP,

United Kingdom

[E-mail: p.t.tucker@swan.ac.uk] 
